\title{
Analysis of Activation Constraints and their Effect on Demand-Side Flexibility Allocations
}

\author{
Kristof Coninx \\ imec-DistriNet \\ KU Leuven \\ 3001 Leuven, Belgium \\ Email: kristof.coninx@cs.kuleuven.be
}

\author{
Geert Deconinck \\ ESAT-ELECTA \\ KU Leuven \\ 3001 Leuven, Belgium \\ Email: geert.deconinck@esat.kuleuven.be
}

\author{
Tom Holvoet \\ imec-DistriNet \\ KU Leuven \\ 3001 Leuven, Belgium \\ Email: tom.holvoet@cs.kuleuven.be
}

\begin{abstract}
Demand-Side Management (DSM) programs in literature and industry alike often enforce constraints on the activation of flexible power provided by customers. While constraining consumption flexibility used in DSM can lower the entry barrier for flexibility providers, the specific constraints used can also have an impact on the efficacy of employing such flexibility. While different problems requiring their own form of consumption flexibility are described in literature, systematic analyses of the impact of activation constraints on such DSM programs are limited. This paper addresses this by analyzing the effects of different activation constraints for power flexibility used in industry, to different problem cases involving renewable production resources. This sensitivity study of activation constraints is performed using real world electricity production and imbalance data for different locations in Belgium. Our work shows that depending on the use case, some constraint dimensions influence the efficiency attained in best case flexibility scheduling while other constraint dimensions do not have any influence in certain other cases. These results are compared against constraint parameters used in flexibility products by system operators today and provide insights into possible improvements of constraint parameters in different use cases.
\end{abstract}

\section{INTRODUCTION}

With the increasing adoption of renewable energy production resources, maintaining a production/consumption balance in the current day electric power grid requires flexibility on both the production and consumption side. Demand-Side Management (DSM) describes techniques for controlling the demand side of the equation to achieve and maintain this balance.

DSM programs can be implemented with different goals in mind. For example, DSM is often considered for flattening peak consumption demand [1] [2]. Improving power system reliability by employing flexible consumption to match intermittent production from Renewable Energy Sources (RES), is also a valid use case of DSM [3]. Other examples exist in both literature [4] and industry [5] of consumption flexibility being harnessed towards a more stable and safe operation of power systems.

Through DSM, consumption flexibility can be employed in different ways to achieve these goals. One category of DSM techniques is called Demand Response (DR). In DR, utilities and system operators indirectly influence the energy demand

978-1-5386-4505-5/18/\$31.00 (C)2018 IEEE IEEE of consumers. This is achieved by offering financial incentives (e.g. tariff reductions) for changes in consumption patterns [6]

Another form of DSM employed in industry is achieved through bilateral agreements between industrial consumers and utilities or system operators [7]. For industrial consumers, bilateral agreements allow consumers to negotiate the form and the availability of their flexibility [8]. Utilities, system operators and other parties that can make use of consumption side flexibility can, in turn, offer standardized flexibility products. Clearly delineated and constrained product offers can lower the entry barrier to potential customers. An integral part of these product are the activation constraints specifying when, how often and for how long flexible power consumption can be used by a contractor. With different ways of employing this constrained flexibility, the specific constraints can have different effects on the global effectiveness of employed flexibility on a case by case basis. Insights into these effects can greatly benefit stakeholders employing consumption flexibility such as Distribution Grid Operators (DSOs), Transmission System Operators (TSOs) and utilities in general.

The presence of systematic evaluations of flexibility activation constraints for industry purposes is limited. This work attempts to remedy that by empirically evaluating the most commonly used flexibility activation constraint dimensions for two different problem cases with real data. A portfolio balancing problem case is compared to a distribution grid congestion case. The portfolio balancing case concerns a Balance Responsible Party (BRP) that balances its portfolio of production and consumption. The production part of this portfolio includes wind production resources. Wind production forecast errors can lead to real time portfolio imbalances that can be offset by consumption flexibility [7] [9]. The distribution grid congestion cases concern DSOs that face grid congestion from wind production during periods with high wind and low consumption. Consumption flexibility can offset overproduction to avoid damaging the infrastructure during congestion events [10]. Both cases have different flexibility needs but can make use of the same consumption flexibility offered by industrial consumers. The effects of flexibility activation constraint parameters and their influence on the effectiveness of scheduling flexible consumption to reduce excess energy are analyzed for both cases. 
Following this introduction, a description of the input data and modeling is given in section II. The analysis approach is described in section III. Analysis results are described in section IV. Finally, the contributions are summarized and described in context of future work in section V.

\section{Model AND DATA}

Small to medium sized companies willing to increase their consumption on demand are modeled to provide consumption flexibility. The amount of flexibility provided at any given time is determined by their flexible power rate. Flexibility providers are realized with flexible power rates drawn from a gamma distribution $\Gamma$ fitted to a confidential industry data set of clients of the tertiary reserve under a dynamic profile (R3DP). This $\Gamma$ distribution $\Gamma(a, r)$ with $a=1.37012, r=677.926$ passes the classical goodness-of-fit such as the Komolgorov-Smirnov [11] and the Anderson-Darling [12] tests for a confidential industry data set of R3DP clients in Belgium. R3DP is a flexibility product offered by the Belgian TSO to distribution grid connected industrial consumers [13].

All flexibility activations are subject to activation constraints. The activation constraint dimensions modeled in this work are inspired by the constraints specified by the R3DP product. These constraints specify an activation duration of two hours with a minimum of twelve hours of inactivity between two consecutive events and a maximum of 40 activations per year. The flexibility modeled in this work will have the same constraint dimensions but with varying parameters as part of a sensitivity analysis. This sensitivity analysis of flexibility activation constraints is performed for different problem cases and real world data from two different locations.

The portfolio balancing case describes the employment of flexible industrial consumers as an alternative to paying imbalance fees to the TSO. When balance responsible parties have portfolios which include sizable amounts of RES, real time balancing becomes more difficult because of the variable nature of these RES. Power production forecasts are an important aspect of minimizing these balances in real time. The imbalances assumed here are caused by wind power production forecast errors between the nomination date of expected production-consumption balance and the actual balance at time of delivery. Imbalance data is generated from proprietary power production time series and forecast error data from literature. These data sets span 12 month periods in 15 minute time periods.

In literature multiple models for wind production forecast error have been proposed based on different underlying distributions. Both the normal distribution and the Cauchy-Lorentz distribution among others have been used to model production forecast errors [14]. In this work we model normally distributed errors with the ERCOT data distribution parameters described in [15]. This imbalance data is compared to imbalance data based on Cauchy distributed production error data artificially created to bear the same total yearly production error volume as the normally distributed data from literature.

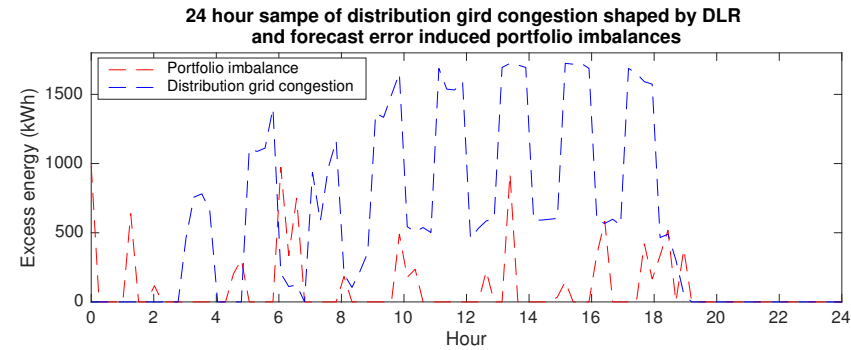

Fig. 1. A random sample of congested energy volumes spanning 24 consecutive hours shows the distinct DLR shaped congestion.

The distribution grid congestion case describes distribution congestion caused by excessive wind power injection. In such cases the cable infrastructure is not rated to cope with excessive currents flowing through the cables at times. The distinction is made between static line rating and Dynamic Line Rating (DLR). Both line rating schemes define which injected power is actually deemed excessive. DLR shows great potential in reducing grid congestion by temporarily allowing excess currents followed by stricter ampacity limits in favor of a universal static ampacity limit [16]. Another useful side effect of using DLR is the window of opportunity it allows in which other flexible power resources can be employed and ramped up to take over when long periods of increased injection are causing problems. Different DLR algorithms produce different congested energy profiles however. In this work two different DLR algorithms are discussed. A first algorithm allows two quarters of an hour of excessive currents up to $30 \%$ more than the static ampacity limit, followed by enforcing a $12.8 \%$ more strict ampacity limit for two quarters hours. A second algorithm allows four quarters of an hour of similar excesses, followed by the same more strict ampacity limit for four quarter hours. Both profiles are discussed in terms of varying activation constraints. Both these cases are discussed for multiple wind power production profiles that differ in the location of the respective wind farms. Concretely, production sites in Zeebrugge and in Antwerp have both provided data for this study detailing a yearly output of $43 \mathrm{GWh}$ and $36 \mathrm{GWh}$ respectively.

Samples of the distribution grid congestion and the portfolio imbalance data is shown in Fig. 1. This sample shows the distinct form of the DLR shaped grid congestion and the sparser portfolio imbalances.

\section{ANALYSIS APPROACH}

The sensitivity analysis focuses on the effects of flexibility activation constraint parameters and their influence on the effectiveness of scheduling flexible consumption to reduce excess energy. Power consumption flexibility is offered by flexibility providers in the form of a fixed number of flexibility activations (c) that are scheduled off line, on fully available historical data. The time series data spans 12 months of excessive energy volumes in 15 minute time periods $t \in\{1, \ldots, 35040\}$. Off line allocation of flexibility provides a view on the upper bound of allocation effectiveness compared to the on line allocation algorithms that correspond to the day to day decision 
making faced by flexibility users when dealing with uncertain production.

The optimal-within-bounds activation schedule is produced using a heuristic local-search algorithm that schedules activations to optimize the actually resolved energy excesses given the following activation constraints:

- An activation event spans a period of $4 * d$ time periods with $d$ representing the activation duration in hours.

- An activation can not start within $4 *(i+d)$ time periods following the start of another activation with $i$ representing the inter-activation time in hours.

- The total number of $c$ activations must be respected.

The heuristic optimization library OptaPlanner [17] is used in the open source simulation framework GridFlex [18]. The first_fit_decreasing construction heuristic from bin packing literature is used to produce an initial allocation schedule [19]. Tabu Search is used as a local search algorithm to further improve the initial allocation schedule [20]. All experiments execute a fixed number of search steps to maintain a fair comparison with varying simulation hardware performance.

Allocation schedules for the different scenarios and combinations of activation constraint parameters are evaluated using the allocative efficiency metric. This metric represents the actual volumes of excess energy that are reduced with the flexibility allocations compared to the total theoretically available flexibility offered by the providers over a 12 month period.

For all analysis scenarios, the relative allocative efficiency metric $\mathcal{E}$ is defined by equation (1) where $C(t)$ represents the congested energy volume function for each 15 minute time period $t$ and $F(t)$ represents the total activated energy volume in the same time periods $\left(C, F:\{1, \ldots, 35040\} \mapsto \mathbb{R}^{+}\right)$. This metric $\mathcal{E}$ is plotted in function of the flexibility activation duration and the minimum inter-activation time parameters.

$$
\mathcal{E}:=\sum_{t \in\{1, \ldots, 35040\}} \frac{\min (C(t), F(t))}{F(t)}
$$

\section{Simulation Results AND Discussion}

This section presents simulation results for the flexibility activation constraint sensitivity analysis. This analysis is performed for the distribution grid congestion case and the portfolio balancing case. All models and data are publicly available in [18]

\section{A. Distribution grid congestion}

When dealing with distribution grid congestion, volumes of excess energy are defined by the electrical current that exceeds the allowed current ratings of cable infrastructure. DLR makes use of the hysteresis effect of current-temperature curves when defining excess energy. We distinguish between the two different line rating algorithms described in section II. The first scenario features a DLR algorithm that allows two quarter hours of excessive current ratings before employing a more strict current rating. A second scenario features an alternative DLR algorithm that allows four quarter hours of

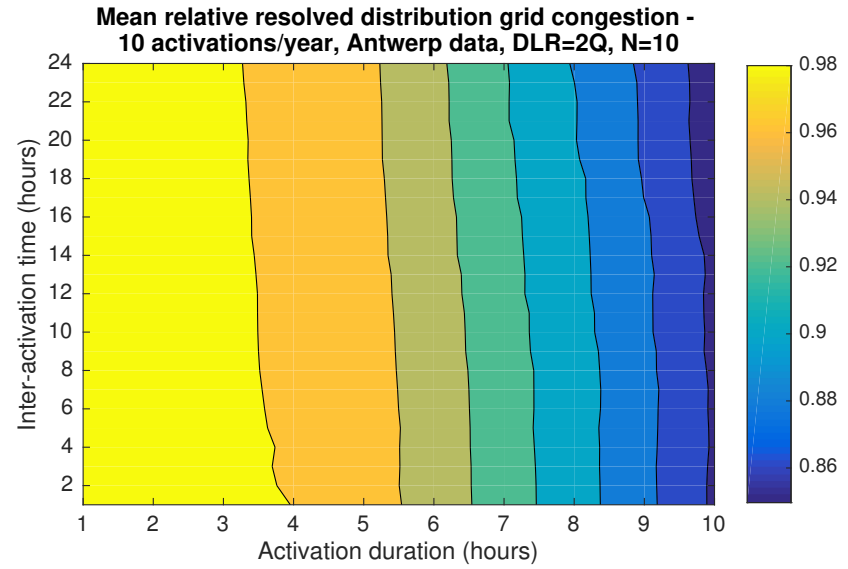

Fig. 2. A gradual decrease of allocative efficiency is shown as the activation duration parameter increases. A small preference for low inter-activation times is noticeable.

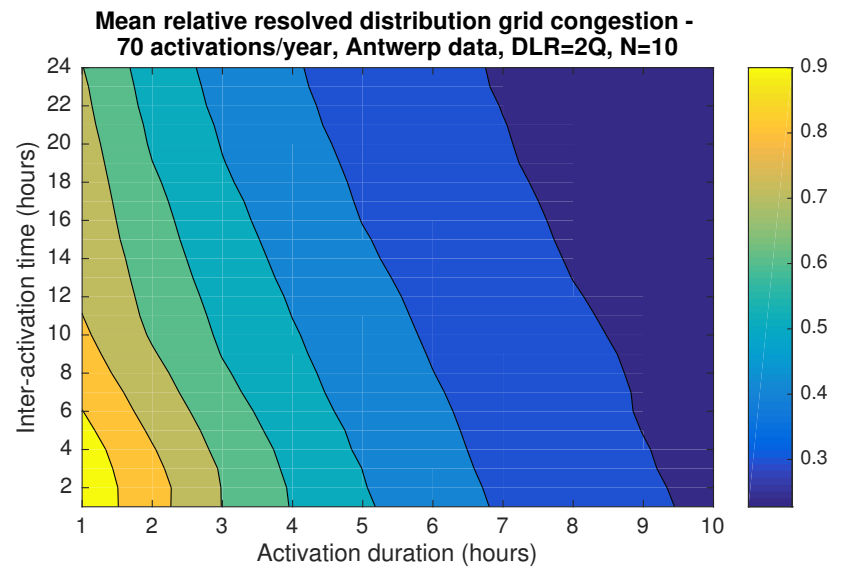

Fig. 3. A gradual decrease of allocative efficiency is shown as the activation duration parameter increases. A preference for low inter-activation times is more pronounced with higher activation events to schedule.

excessive current ratings before employing the same strict rating.

1) Influence of dynamic line rating parameters: Results in Fig. 2 shows the relative allocative efficiency metric for the first scenario using the Antwerp data set. These results show a gradual decrease in relative efficiency as the activation duration increases. A small preference for smaller inter-activation times is also noticeable. This effect becomes more noticeable when more activation events need to be scheduled, which is shown in Fig 3.

For the second scenario, results shown in Fig. 4 show a similar decrease in efficiency with increasing activation durations in general. One important difference in these results is the markable increase in efficiency relative to the observed results from the first scenario when using activation durations of odd hours. Results for the second scenario using the Zeebrugge data set are shown in Fig. 5. Comparing these results with the Antwerp data set shows that the inter-activation time parameter bears no effect on the allocative efficiency metric by itself in this case. A general decrease of allocative efficiency as the 


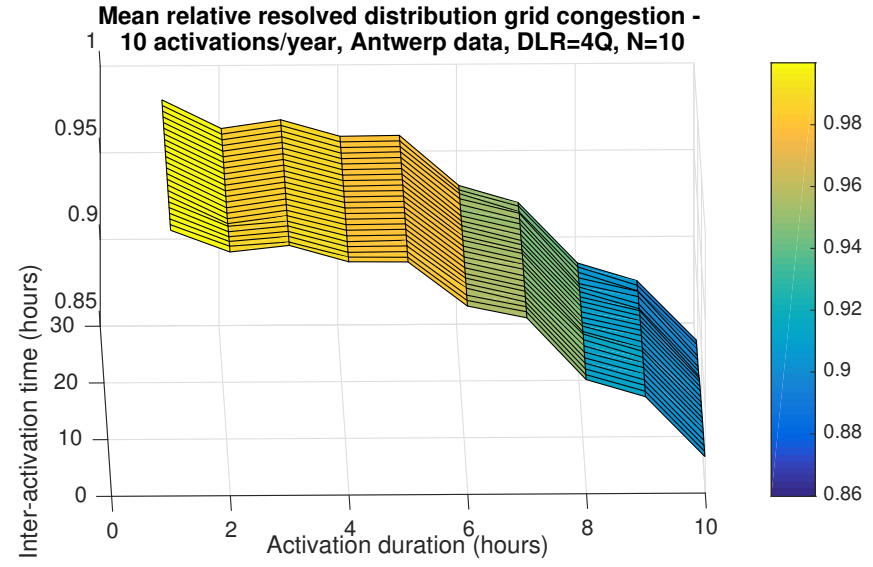

Fig. 4. A general benefit for odd activation durations over even durations is noticeable.

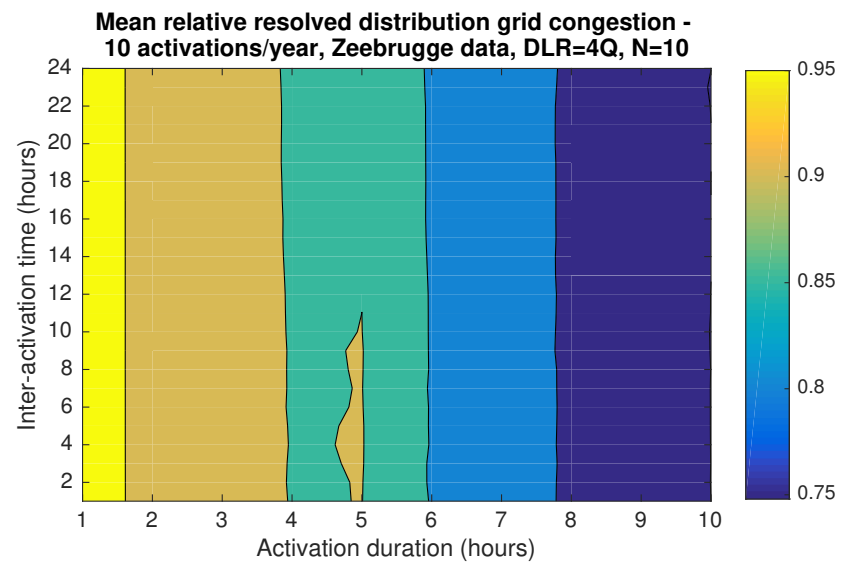

Fig. 5. The preference of lower inter-activation times is less pronounced in the Zeebrugge data set. These results show similar benefits for odd activation durations as the results in Fig.4

activation duration increases is noticeable however. Similar to the second scenario of the Antwerp dataset, odd hours of activation durations yield greater or equal allocation efficiency than using smaller but even hours of activation durations.

Intuitively, allocating activation events with even hour durations to congestion which oscillates hourly between high and low values results in the best case in allocations that have at least $50 \%$ of the time spanning the low values. Activation events with odd hour can both start and end on the high values, thereby possibly yielding higher efficiencies. This is supported analytically by first examining a stylized congestion function that produces a similar congestion profile as DLR algorithms that allow $k$ quarter hours of high cable ratings followed by $k$ quarter hours of lower ratings. Function (2) represents such a binary step function.

$$
C^{*}(x)=1-\left\lfloor\frac{x}{k}\right\rfloor \bmod 2
$$

The relative amount of congestion $r$ that can be resolved by an activation of $d$ hours is given in (3).

$$
r=\frac{1}{d} \int_{0}^{4 d} C^{*}(x) d x
$$

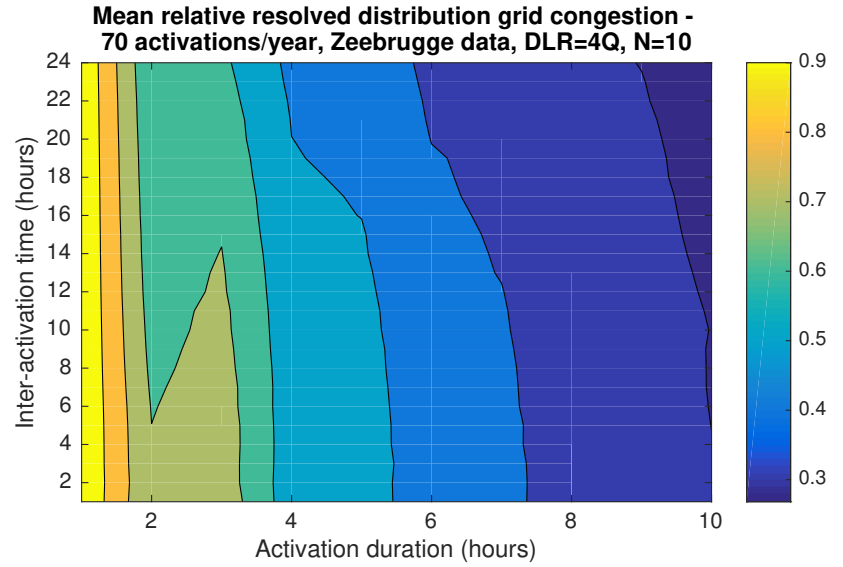

Fig. 6. Scheduling 70 allocation events causes the allocative efficiency to decrease when using high duration activations, when compared to lower numbers of allocations to schedule.

For $k=2$, equation (3) yields a constant $r=2$ for all values of $d \in \mathbb{N}^{+}$. For $k=4$, equation (3) yields a case distinction between even and odd values of $d$. This case distinction resulting from evaluating (3) is given in (4) and shows a similar limit behavior as for $k=2$ but with possible higher fractions of resolved congestion for smaller odd values of $d$. This concurs with the results shown in Fig. 4 and Fig. 5.

$$
r= \begin{cases}2, & \frac{d}{2} \in \mathbb{N}^{+} \\ \frac{4\left\lceil\frac{d}{2}\right\rceil}{d}, & \frac{d}{2} \notin \mathbb{N}^{+}\end{cases}
$$

2) Influence of offered flexibility volumes: The least constrained activations to schedule are those activations that last only an hour and can be activated again with only an hour of down time between two consecutive activations. In all scenarios shown, those conditions offer the highest allocative efficiency. When varying the number of activations that have to be scheduled at once, these conditions remain dominant in their effectiveness. Even when increasing the number of activations that have to be scheduled eightfold, the relative allocative efficiency metric attains higher than $90 \%$ efficiency in these conditions. When looking at activation durations that last longer, the allocation efficiency decreases when the number of allocations to schedule increases. This is apparent when comparing Fig. 2 with Fig. 3 for the Antwerp data set. But also comparing Fig. 5 with Fig. 6 shows a similar trend.

When keeping to a fixed number of allocations and varying the activation durations within a scenario, the total amount of flexibility offered by a flexibility provider varies also. To avoid presenting a skewed view of optimal constraints for providers willing to provide a set amount of flexibility, Fig. 7 and Fig. 8 show results in terms of allocative efficiency for which the number of allocation events to schedule is scaled in function of the activation duration as to keep total amount of flexibility offered by providers constant.

Results for the Antwerp data shown in Fig. 7 show unsurprisingly a preference for highly flexible activations with short activation durations and short inter-activation times. It is also 
Mean relative resolved distribution grid congestion equal total activation energy, Antwerp data, $D L R=4 Q, N=20$

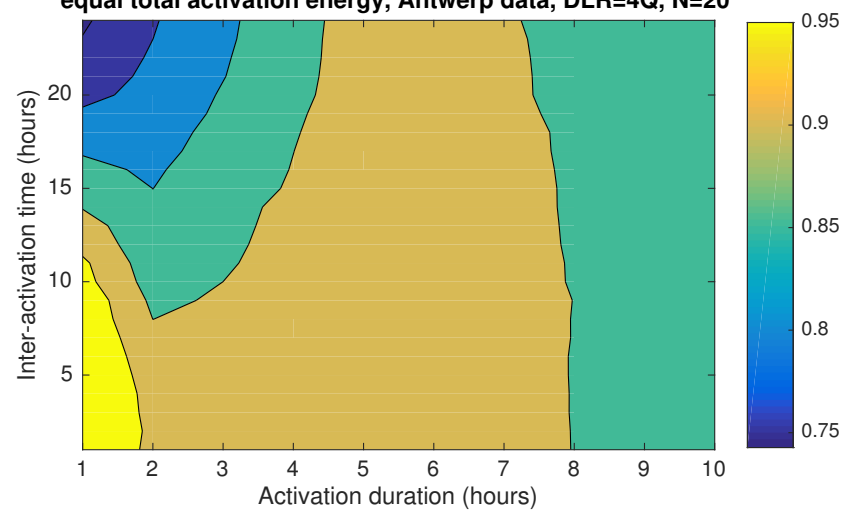

Fig. 7. When the total flexibility offered is assumed constant over varying activation constraints then a clear preference for minimally constrained flex is noticeable.

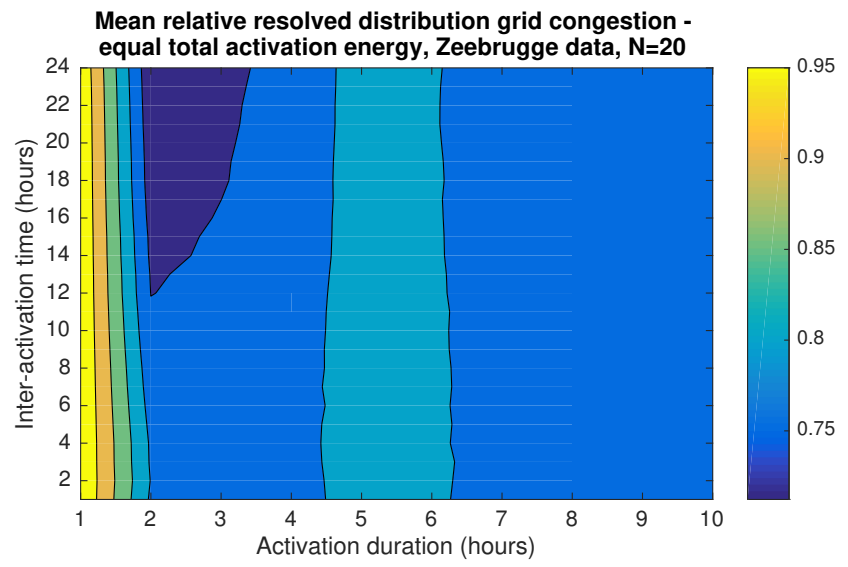

Fig. 8. When the total flexibility offered is assumed constant over varying activation constraints then a clear preference for minimally constrained flex is noticeable. A local maximum can also be found with activation durations of 5-6 hours

noticeable that short activation durations need to be paired with short inter-activation times to produce relatively effective allocations. Increasing the activation duration consecutively allows for higher inter-activation times as well without losing significant efficiency in flexibility allocations.

For the Zeebrugge data shown in Fig. 8 a general disinterest in the inter-activation time is observed for all activation durations except durations ranging from two to four hours. Furthermore, short activation durations of one hour are preferred to produce optimally efficient allocations. If one hour activation durations are not feasibly, however, a local optimum can be found for activation durations ranging from five to six hours.

It can also be noted that in all simulation results, the parameters used by the R3DP product (40 activations of two hours with an inter-activation time of 12 hours), can be improved upon to provide more efficient activation schedules.

\section{B. Portfolio balancing}

The difference between normally distributed forecast errors and Cauchy distributed errors is that, in the latter case, higher

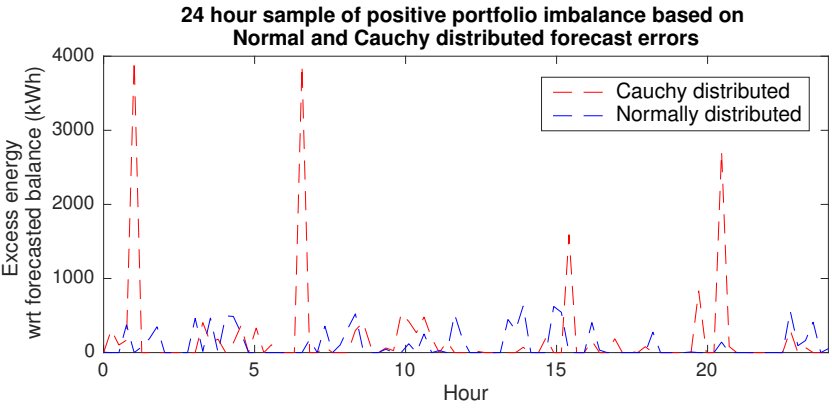

Fig. 9. This 24 hour sample of the normally and Cauchy distributed forecast errors shows a difference in peak error levels and the frequency of the occurring peaks.

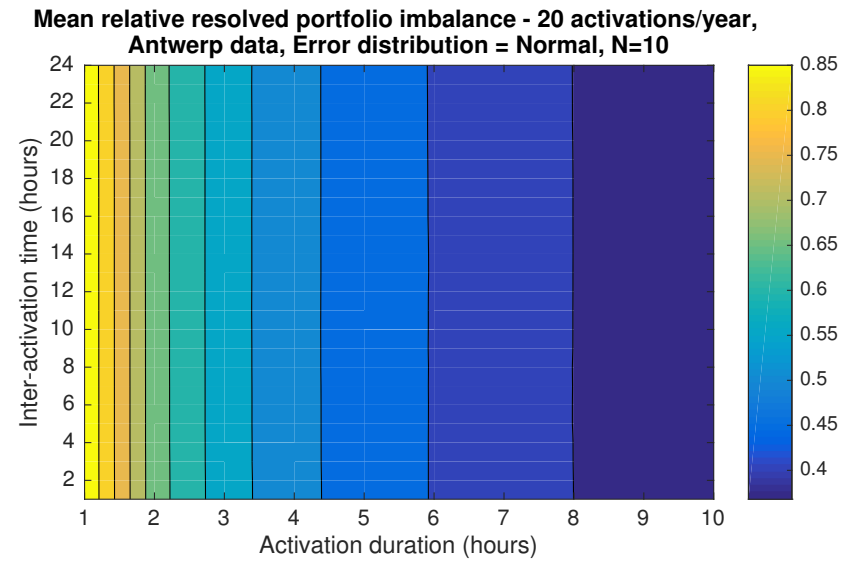

Fig. 10. Relative resolved portfolio imbalance for the Antwerp data shows that the inter activation time parameter has no influence on the efficiency of flexibility activation.

error peaks can be observed, but at a lower frequency than the normally distributed error peaks. Figure 9 shows a sample period for each distribution.

Simulation results from allocating flexibility under different activation constraints are shown in Fig. 10 for the normally distributed forecast errors and in Fig. 11 for the Cauchy distributed errors using the Antwerp power production data. Literature has indicated that the use of different forecast error distributions can yield different results. No such discernible differences in trends were observed in these results. Results using the normal distribution show an overall small $(<5 \%)$ increase in allocative efficiency over the Cauchy distributed errors. Results from the Zeebrugge production data set show trends similar to the Antwerp data.

Compared to the problem cases discussed in section IV-A, in portfolio balancing cases, the inter-activation time parameter of the activation constraints has no influence on the allocative efficiency of these activations. Forecast errors are less subject to seasonal variations than the distribution grid congestion case because of the different origin of the error. This limited seasonality in the problem data means that for a limited amount of activations to schedule, the probability of finding volumes of excessive energy for an activation that does not conflict with other activations is high enough for the inter-activation time 


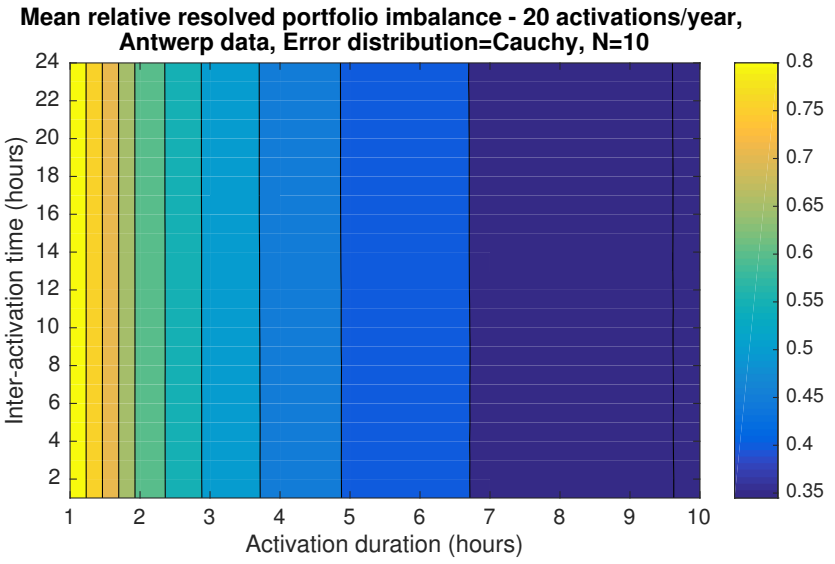

Fig. 11. The results using the Cauchy distributed forecast error shows no difference in trend when compared to normal distributions.

parameter not to matter.

\section{CONCLUSION}

Maintaining a real time production/consumption balance is challenging in light of increased adoption of renewable energy sources. Bilateral contracts between utilities and flexible consumers can regulate the use of consumption flexibility to maintain this balance in real time. In this work we perform a sensitivity analysis of flexibility activation constraint parameters when used to schedule flexibility activations in two problem cases for two locations in Belgium. This analysis is performed for the off line scheduling of consumption flexibility activations based on real world data spanning 12 consecutive months.

Simulation results show that constraining the required down time that follows an activation of consumption flexibility can only influence the efficiency of flexibility scheduling in distribution grid congestion cases and not in portfolio balancing cases. Results also show that whether a normal or a Cauchy distribution is used to model forecast errors has no effect on the efficiency of flexibility scheduling. Finally, results also indicate that when DLR is used to shape congestion in distribution grid congestion cases, there is a clear relation between the DLR algorithm and the activation duration parameters used. Results presented in this work can offer insights into how the design of flexibility products offered by system operators and other parties requiring flexibility can be improved upon in terms of activation constraints. For distribution grid congestion cases specifically, more efficient flexibility activations are possible when activation duration and inter-activation times can be lowered, compared to R3DP.

Future work into the use of other DLR algorithms in the sensitivity study can prove useful in providing a better understanding of the effects of DLR on flexibility activation constraints. Expanding this sensitivity analysis to on line allocation mechanisms as well to gain understanding of optimal constraints to use when dealing with uncertain future production excesses is another opportunity for future work.

\section{ACKNOWLEDGMENT}

This research is partially funded by the Research Fund KU Leuven.

\section{REFERENCES}

[1] M. Uddin, M. F. Romlie, M. F. Abdullah, S. Abd Halim, A. H. Abu Bakar, and T. Chia Kwang, "A review on peak load shaving strategies," Renewable and Sustainable Energy Reviews, nov 2017.

[2] D. Lizondo, P. Araujo, A. Will, and S. Rodriguez, "Multiagent Model for Distributed Peak Shaving System with Demand-Side Management Approach," in 2017 First IEEE International Conference on Robotic Computing (IRC). IEEE, apr 2017, pp. 352-357.

[3] M. Kushler, E. Vine, and D. York, "Using energy efficiency to help address electric systems reliability: an initial examination of 2001 experience," Energy, vol. 28, no. 4, pp. 303-317, 2003.

[4] M. Paulus and F. Borggrefe, "The potential of demand-side management in energy-intensive industries for electricity markets in Germany," Applied Energy, vol. 88, no. 2, pp. 432-441, feb 2011.

[5] F. Boshell and O. Veloza, "Review of developed demand side management programs including different concepts and their results," in 2008 IEEE/PES Transmission and Distribution Conference and Exposition: Latin America. IEEE, aug 2008, pp. 1-7.

[6] R. Deng, Z. Yang, M. Y. Chow, and J. Chen, "A survey on demand response in smart grids: Mathematical models and approaches," IEEE Transactions on Industrial Informatics, vol. 11, no. 3, pp. 570-582, 2015

[7] K. Heussen, S. You, B. Biegel, L. H. Hansen, and K. B. Andersen, "Indirect control for demand side management - A conceptual introduction," in 2012 3rd IEEE PES Innovative Smart Grid Technologies Europe (ISGT Europe). IEEE, oct 2012, pp. 1-8.

[8] F. Lopes, C. Ilco, and J. Sousa, "Bilateral negotiation in energy markets Strategies for promoting demand response," in 2013 10th International Conference on the European Energy Market (EEM). IEEE, may 2013, pp. 1-6.

[9] K. Coninx and T. Holvoet, "Darwin in Smart Power Grids Evolutionary Game Theory for Analyzing Self-Organization in DemandSide Aggregation," in 2015 IEEE 9th International Conference on Self-Adaptive and Self-Organizing Systems, vol. 2015-Octob. Boston: IEEE, sep 2015, pp. 101-110.

[10] K. Coninx, M. Moradzadeh, and T. Holvoet, "Combining DSM and storage to alleviate current congestion in distribution grids," in 2016 IEEE PES Innovative Smart Grid Technologies Conference Europe (ISGT-Europe). Ljubljana: IEEE, oct 2016, pp. 1-6.

[11] F. J. Massey Jr, "The Kolmogorov-Smirnov test for goodness of fit," Journal of the American statistical Association, vol. 46, no. 253, pp. 68-78, 1951.

[12] F. W. Scholz and M. A. Stephens, "K-sample Anderson-Darling tests," Journal of the American Statistical Association, vol. 82, no. 399, pp. 918-924, 1987.

[13] E. cvba, "Flexibiliteit R3DP," 2018. [Online]. Available: http://www.eandis.be/nl/klant/aansluitingen/meer-overaansluitingen/ flexibiliteit-r3dp

[14] B.-M. Hodge, E. Ela, and M. Milligan, "The distribution of wind power forecast errors from operational systems," in 10th International Workshop on Large-scale Integration of Wind Power, 2011.

[15] B.-M. Hodge, D. Lew, M. Milligan, H. Holttinen, S. Sillanpää, E. GómezLázaro, R. Scharff, L. Söder, X. G. Larsén, G. Giebel, H. Holttinen, S. Sillanpää, R. Scharff, L. Söder, D. Flynn, and J. Dobschinski, "Wind power forecasting error distributions: An international comparison," in 11th International Workshop on Large-Scale Integration of Wind Power, Lisbon, 2012, pp. 1-9.

[16] A. K. Kazerooni, J. Mutale, M. Perry, S. Venkatesan, and D. Morrice, "Dynamic thermal rating application to facilitate wind energy integration," in 2011 IEEE Trondheim PowerTech. IEEE, jun 2011, pp. 1-7.

[17] G. De Smet, "OptaPlanner User Guide," 2017. [Online]. Available: http://www.optaplanner.org

[18] K. Coninx, "GridFlex Simulator," 2017. [Online]. Available: https: //github.com/KrisC369/GridFlex-Simulator

[19] L. T. Kou and G. Markowsky, "Multidimensional Bin Packing Algorithms," IBM Journal of Research and Development, vol. 21, no. 5, pp 443-448, 1977.

[20] L. D. Ha, S. Ploix, E. Zamai, and M. Jacomino, "Tabu search for the optimization of household energy consumption," in 2006 IEEE International Conference on Information Reuse \& Integration, 2006, pp. 86-92. 